\title{
SURGICAL TREATMENT OF ANTERIOR SEPTAL DEVIATIONS
}

\author{
David Núñez-Fernández, Jan Vokurka \\ Department of Otorhinolaryngology, Charles University, Faculty of Medicine and Teaching Hospital, Hradec Králové; \\ (Head: doc. MUDr. J. Vokurka, CSc.)

\begin{abstract}
Summary: Anterior septal deviations are very difficult to handle because their location causes valvular obstruction, and any small residual deviation can cause a high degree of obstruction. The commonly performed submucous resection is not a suitable technique to handle this deformity. The maxilla-premaxilla technique offers a better approach. The surgical technique for anterior septal deviation differs from the standard septoplasty in several steps. A modified technique to treat this particular pathology is described in detail. This technique is highly reliable, and simplifies the access to a difficult-to-handle deformity.
\end{abstract}

Key words: Septoplasty; Anterior nasal septum deviation surgery; Caudal septum deviation; Nasal valve surgery operative technique

\section{Introduction}

Anterior septal deviations are those localized in the vestibular and valvular areas of the nose (Cottle's areas II and III). These deviations, usually caused by trauma, produce a high degree of obstruction. Due to their location, anterior septal deviations produce valvular collapse. They also produce further deformities in the septum, including ipsilateral subluxation and posterior spurs (Fig. 1), which make its surgical management more complicated, and increase the risk of complications, such as septal perforation.

The major difference between the anterior septal deviations and the more common deviations in the turbinal area of the septum, are the difficulties in management and the predictability of the final result.

Anterior septal deviations are not suitable to be handled with submucous resection. Nothing short of a complete septoplasty (the maxilla-premaxilla approach $(2,4)$ ) with valvular surgery will resolve the deformity. It is not uncommon to see patients with ASDs, who have undergone submucous resection, where in the most prominent part of the deviation was resected, but the superior part was left intact, with a persistent valvular collapse. Although the rest of the septum is straight, the patient continues to complain of nasal obstruction.

Another common mistake is to resect the caudal border of the septal cartilage to obtain a straight septum (5). This will, over time, cause collumelar retraction, and still leaves the problem of the subluxation and spurs in the posterior part of the septum.

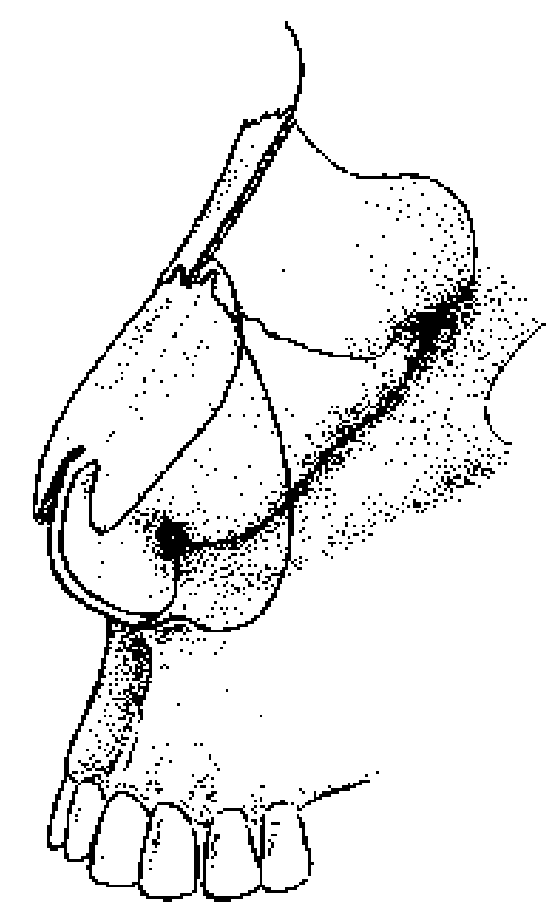

Fig. 1: Preoperative view, notice deviations in valvular area, and in anterior and posterior chondrovomerine articulation.

\section{Material and Methods}

The authors have found that the following steps are necessary to secure good results in this surgery (Fig. 2). 

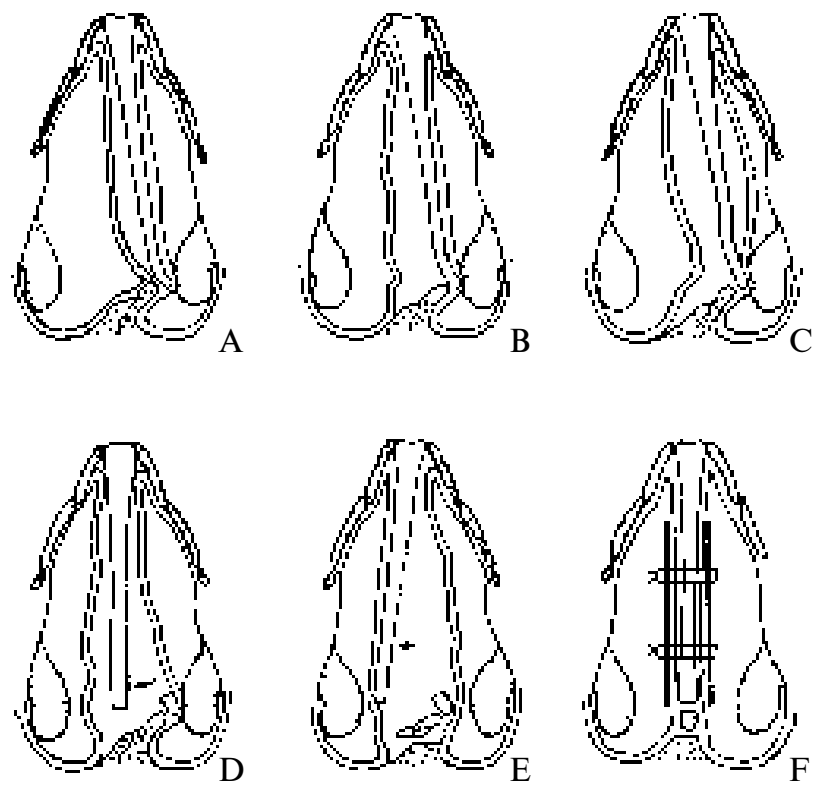

Fig. 2:

A) Dissection of anterior tunnel on the concave side.

B) Careful dissection of inferior tunnel on the concave side and connection with the anterior tunnel.

C) Dissection of anterior tunnel on the convex side without dissecting the most prominent part of the deviation.

D) After cutting the cartilage proximal to the deviation, the superior part is moved to the other side to allow visualization of the deviation.

E) The bony part of the deviation is excised with an osteotome and the most prominent part of the deviation is dissected from the mucosa.

F) All remaining obstructive parts of the deviation are removed. The superior part of the cartilage is moved towards the center and fragments of cartilage or bone can be repositioned. Silastic splints are sutured to hold the mucosal flaps attached to the septum and act like a cast.

1) Right hemitransfixion incision and dissection of tunnels in concave side: We recommend to dissect first the concave side of the septum, because in this side the mucosa is under less tension and is more difficult to fenestrate (Fig. 2A). It is usually easier to dissect the inferior tunnel on this side (Fig. 2B).

2) Dissection of anterior tunnel in convex side: This part of the surgery is very difficult and care must be taken not to perforate the mucosa on this side. In extremely large deviations, it is recommended to divide them, making a vertical cut in the angle formed by the anterior and posterior parts of the cartilaginous deviation. Care should be taken not to divide it completely to the roof, to avoid a difficult reconstruction. This will leave the anterior segment almost free, and will avoid complete displacement. An incision over all the chondrovomeri- ne articulation will make the dissection easier. Under good visualization, the posterior part of the deviation is gently separated from the mucosa, towards the concave side (Fig. 2C).

3) Dissection of inferior tunnel of the convex side: The best approach is through the exposure of the nasal spine, and the separation of the mucosa from the bony part of the spur. Sometimes, however, this part lies low over the floor of the nose, or there is excessive tension on the mucosa. In these cases, we cut the most prominent part of the spur or the complete bony deviation with an osteotome ( $2 \mathrm{~mm}$ osteotome). This reduces the tension in the area, and makes it easier to separate the fragment from the mucosal flap and to continue the dissection of the inferior tunnel (Fig. 2D).

4) Resection of deviation: Once exposed, a small strip is excised from the inferior border of the septal cartilage, separated from the vomer and removed. The articulation between septal cartilage and perpendicular plate of ethmoids (PPE) is also divided. This leaves the septal cartilage almost free of tension. The superior part of cartilage is mobilized towards the other side. The bony part of the deviation is now fully visible. The deviation is resected with osteotome or scissors, depending on the size of the deviation and the surgeon's preference (Fig. 2E) .

5) Verification of the valvular area patency: This frequently overlooked step is necessary to correct any deviation found in the nasal valve and the roof of the nose. These deviations are frequently found in two places:

-I) The union of the septal cartilage with the PPE: To correct this, it is necessary to resect the bony part of the articulation, and to free the posterior border of the septal cartilage so it can be moved back to the center. If this is still not possible, is necessary to make a horizontal incision in the convex side of the cartilage, near the roof of the nose, in order to add flexibility and allow movement to the cartilage.

- II) The valvular area, where the most anterior part of the deviation was found: This should be the last portion to be modified, because it is necessary to suture the inferior part of the septum to the nasal spine, in order to avoid the complete displacement of the caudal part of the septum. Once the inferior portion of the septum is sutured, it is possible to resect and reconfigure the remaining superior deviation. Commonly, it is necessary to modify the nasal valve. If this is the case, the septum should be separated from the upper lateral cartilage and the nasal valve trimmed as in a valvuloplasty. A spreader graft can be used as a batten to straighten this area and open the nasal valve.

- The final result should look like Fig. 3. 


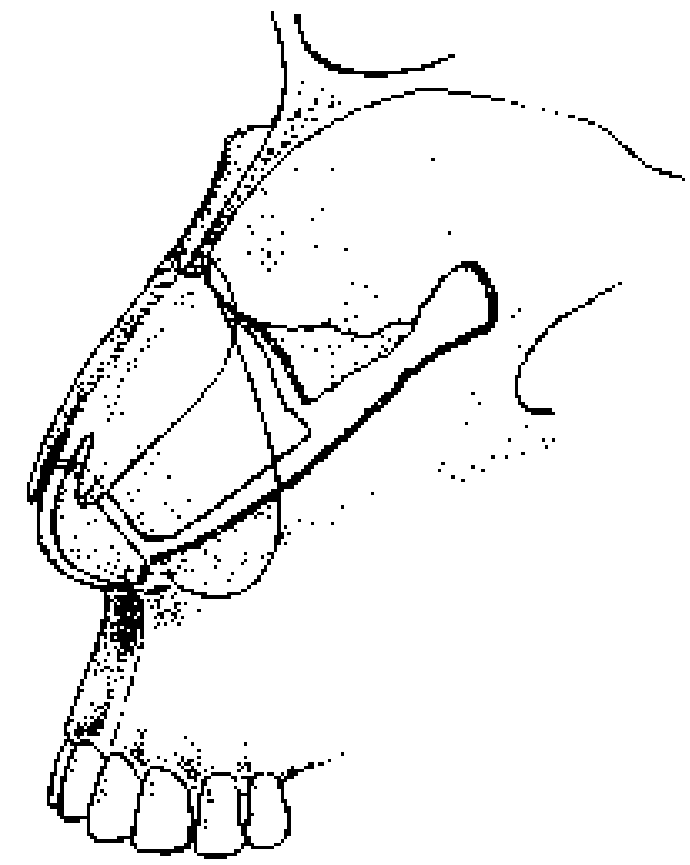

Fig. 3: Postoperative view: the caudal fragment should be sutured to the nasal spine and to the posterior fragment of the septal cartilage.

6) If there is a large fenestration of the mucosa in the convex side of the deviation, it is better to close it with two or three sutures.

7) If a hypertrophic inferior turbinate in the concave side exists, this is a good time to make a partial turbinectomy. The better and longest lasting results are achieved by conservative resection of the excessive mucosa and bone.

8) Reposition of bony or cartilaginous fragments is recommended when large parts of the septum has been excised. The hemitransfixion is sutured with catgut 4-0. After all incisions are sutured, the septum is held with silastic splints (Fig. 2F). Vaseline or packing covered with antibiotic ointment is applied to both nasal cavities. If inferior turbinectomy was performed, it is necessary to compress the wounded tissue to avoid bleeding. This packing is removed after two days.

The patient may be discharged from the hospital the same day of surgery, depending upon recovery. If the patient lives far from the hospital, it is better to wait five days to discharge, after the removal of the splints.

It is advisable to give the patient an explanatory leaflet about the surgery which include what he should, and what shouldn't do, possible complications, and how to handle them.

The technique described above is very reliable in the management of area II complex deviations. The improvement in airway patency is dependent on the reconstruction of the superior part of the deviation and the nasal valve.
These are the most neglected areas in this surgery. In these areas, a small deviation can produce subjective and objective (rhinomanometric) obstruction, even if the rest of the septum is straight.

\section{Discussion}

Many authors agree in the technical difficulty to correct anterior septal deviations $(1,3,6)$. Area II deviations were very difficult to handle before the advent of the maxilla-premaxilla approach described by Cottle. This technique allows the surgeon to have a complete view of the septum, thereby providing the ability to reconstruct it, rather than simply excising it. This approach is more popular in America than in Europe, where it is considered complicated and time-consuming. Submucous resection is still the preferred technique in Europe, even though it has more complications, and is unable to handle anterior deviations or complex deformities of the nose.

Submucous resection is indicated in some septal pathologies, particularly for inferior spurs due to subluxation. In these cases a classical Killian incision is enough to obtain an adequate exposure of the deviation. The maxilla - premaxilla approach is better suited for anterior deviations (area I, and II) or complex deviations which require the complete exposure of the septum prior to its reconstruction. This technique can be easily mastered by the beginning surgeon, if used frequently enough.

The most important part of the surgery is finding the right plane of dissection and the authors want to emphasize this. This plane is sometimes difficult to find, particularly in post-traumatic cases. Dissecting in this plane helps to avoid undesired fenestrations of the mucosa, and reduces the risk of a septal perforation.

Also important is the repositioning of the fragments of cartilage and bone. This is important when extensive resection was made and we have large areas of both mucosa layers in direct contact. The fragments can be straightened with bisturi, with a bone crusher or with a morselizer. It isn't necessary to replace all fragments, is better to put the larger ones to give some rigidity to the septum and avoid the well-known complications of the lax septum.

\section{Conclusion}

A technique has been described to treat anterior septal deviations. This technique is easily reproducible and highly reliable. In this way, it is possible to restore the normal anatomy of the nasal valve allowing a good breathing function.

\section{Acknowledgments}

The authors would like to thank PhDr. Josef Bavor from the Department of Anatomy of the Charles University, Faculty of Medicine in Hradec Králové, for his nice work in the illustrations. 


\section{References}

1. Beeson WH. Septal surgery. In: Daniel, RK, ed. Aesthetic plastic surgery: Rhinoplasty. Boston-Toronto-London, Little, Brown and Company, 1993:595-611.

2. Cottle MH. The maxilla -premaxilla approach to extensive nasal septal surgery. Arch Otolaryngol 1958;68:301-13.

3. Karmer S, Churukian M. High septal hemitransfixion for the correction of caudal septal deformities. Laryngoscope 1984;94:391-4.

4. Klaff DD. The surgical anatomy of the antero-caudal portion of the nasal septum: A study of the area of the premaxilla. Laryngoscope 1956;66:995-1020.

5. Metzenbaum M. Replacement of the lower end of the dislocated septal cartilage versus submucous resection of the dislocated end of the septal cartilage. Arch Otolaryngol 1929;9:282-96
6. Stal S. Septal deviation and correction of the crooked nose. In: Daniel RK, ed. Aesthetic plastic surgery: Rhinoplasty. Boston-Toronto-London, Little, Brown and Company, 1993:595-611.

Submitted May 1998.

Accepted June 1998.

MUDr. David Núñez-Fernández, Department of Otorhinolaryngology, Charles University, Faculty of Medicine and Teaching Hospital, 50005 Hradec Králové, Czech Republic. 